\title{
Reexamining the Age-Misconduct Curve: Interaction Effects of Sex and Race/Ethnicity*
}

\author{
Yaş-Kural İhlali Ĕgrisini Yeniden Keşfetmek: Cinsiyet ve Irk/Etnik \\ Köken Etkileşim Etkileri
}

\section{Colby L. VALENTINE ${ }^{1 \oplus}$}

${ }^{1}$ Assist. Prof., Domincan College, Division of Social Sciences, Orangeburg, New York - USA

\section{ABSTRACT}

Numerous studies have focused on age as one of the most robust correlates of prison misconduct, with younger inmates more likely to commit disciplinary infractions compared to their older counterparts. However, less empirical attention has been paid to the conditioning effects of additional demographic variables such as sex or race/ethnicity on the agemisconduct relationship. The current study, thus, further extends prior research on age and prison misconduct by examining potential interaction effects between age and sex (i.e., male and female), and separately, between age and race/ethnicity (i.e., non-Hispanic white, non-Hispanic black, and Hispanic). Negative binominal regression models are used to examine 137,552 inmates admitted to state prison in Florida from 1995-2000. Analyses indicate that overall, there are no substantial differences between males and females or between racial/ethnic categories when examining the relationship between age and prison misconduct. However, the youngest offenders warrant some attention for their visible differences in levels of misconduct for both males and females and for Hispanic, black, and white inmates. Research and policy implications are discussed regarding the potential variation in the age-misconduct curve, especially for younger inmates.

Keywords: Prison misconduct, race/ethnicity, sex

"The following manuscript is based on the doctoral dissertation: Valentine, Colby Lynne 'Unraveling the age, prison misconduct, and recidivism relationship' (2012) Retrieved from Electronic theses, treatises and dissertations. Paper 5243. 


\section{Introduction}

Several studies have examined the various individual characteristics that may be associated with prison misconduct. For instance, scholars have found a negative relationship between age and misconduct in studies of both male and female inmates (Camp, Gaes, Langan, \& Saylor, 2003; Craddock, 1996; DeLisi, 2003; Gover, Pérez, \& Jennings, 2008; Jiang \& Winfree, 2006; Steiner, Butler, \& Ellison, 2014; Valentine, Mears, \& Bales, 2015). Further, an inmate's race or ethnicity has also been associated with various types of misconduct (Berg \& DeLisi, 2006; DeLisi, 2003; DeLisi, Trulson, Marquart, Drury, \& Kosloski, 2011; Gover et al., 2008; Harer \& Steffensmeier, 1996; Steiner \& Wooldredge, 2009a). However, much less empirical attention has been paid to the conditioning effects of either sex or, separately race/ethnicity, on the age-misconduct relationship. If such variation does exist, then this information may provide insight into future correctional policies, such as intake assessments or security placement, for controlling prison order among specific types of inmates.

The current study advances the scholarship on the effect of sex on prison misconduct and also the effect of race on prison misconduct in several ways. First, little research exists that examines the joint effect of both age and sex and also age and race/ethnicity on misconduct. Therefore, the current study examines if agemisconduct curves differ according to either sex or race/ethnicity. Second, the Florida Department of Corrections is the third largest state correctional system in the United States and allows for the investigation of a large admissions cohort to systematically examine granular age dummy variables to predict the precise age-misconduct curves for both males and females and also for Hispanic, non-Hispanic Black, and nonHispanic white inmates. Third, this data also provides the opportunity to examine a variety of misconduct types including: general, violent, property, drug, and disorderly.

\section{Literature Review}

\subsection{Age, Sex, and Prison Misconduct}

Numerous studies have examined sex differences in factors, such as age, contributing to prison misconduct (Berg \& DeLisi, 2006; Camp et al., 2003; Craddock 1996; Gover, Pérez, \& Jennings, 2008; Jiang \& Winfree, 2006; McClellan, 1994); however, most studies focus on male only samples (Cunningham \& Sorensen, 2007; DeLisi et al., 2011; Harer \& Steffensmeier, 1996; Kuanliang, Sorensen, \& Cunningham, 2008; McReynolds \& Wasserman, 2008; McShane \& Williams, 1989; 
Morris et al., 2010; Steiner \& Wooldredge, 2008; Tasca, Griffin, \& Rodriguez, 2010) and a small number examine female only samples (Blackburn \& Trulson, 2010; Houser, Belenko, \& Brennan, 2012; Pollock, Mullings, \& Crouch, 2006; Steiner \& Wooldredge, 2009b). Much less empirical attention has been paid to the sex differences in prison misconduct and especially the conditioning effect sex may have on the age-misconduct relationship.

The limited research that has examined sex differences confirms that differences do exist; however, the findings are mixed. Prior work has attempted to model sex differences in two ways: 1) analyze the effect of sex in one model, or 2) analyze separate models for males and females. Of the studies that controlled for sex, some found male inmates more likely to be involved with various types of prison misconduct (Kuanliang \& Sorenson, 2008; Sorensen \& Cunningham, 2010), one found being female increased the probability of severe misconduct violations (Cao, Zhao, \& Dine, 1997), and others found no statistically significant sex differences for major or violent prison misconduct (DeLisi, 2003; DeLisi \& Munoz, 2003).

A limited number of studies have attempted to examine sex differences in factors that influence prison misconduct by examining separate regression models for both males and females (Berg \& DeLisi, 2006; Craddock, 1996; Gover et al., 2008). For example, Gover et al. (2008) examined the effect of importation and deportation measures on institutional misconduct for both males and females. Specifically focusing on age, they found in the female model, being older was significantly related to fewer infractions. However, there was no statistically significant relationship between age and misconduct for males. Berg and DeLisi (2006) also analyzed separate regression models for males and females to examine the effects of various correlates of prison misconduct. The results found that age was not significantly related to prison violence for males or females in either model.

These studies have made noteworthy steps toward expanding the prison misconduct literature by focusing on the independent effects of age, and other covariates, on prison misconduct for both males and females; however, neither study attempted to measure the interactive or nonadditive effects of age and sex. For that reason, there still remains much unknown about the conditioning effect of sex on the age-misconduct relationship.

Whatever drives the differences in misconduct among males and females, they may be more noticeable among late adolescent and young adult inmates for several 
reasons. First, the juvenile justice literature suggests that young inmates, especially males, express concern to be accepted by others and thus engage in higher levels of misconduct to prove their toughness and masculinity (Bishop \& Frazier, 2000). In addition, the process in which inmates cope with the deprivations of prison may differ according to sex. For example, males feel a greater amount of powerlessness and lack certain types of social support while incarcerated which may lead to a more difficult adjustment resulting in a higher rate of misconduct infractions compared to females (Jiang \& Winfree, 2006; Zingraff, 1980). Further, some scholars argue that low self-control may be related to misconduct among male inmates (Gover, Pérez, \& Jennings, 2008; Hochstetler \& DeLisi, 2005). Thus, one would expect younger, male inmates to engage in more misconduct compared to younger, female inmates.

Notwithstanding the aforementioned research, Valentine and colleagues (2015) illustrated a curvilinear relationship between age and prison misconduct as well as predicted a negative relationship between male inmates and general, violent, property, and disorderly misconduct. Conversely, being male was positively related to drug misconduct. Thus, the current study extends this research, as well as others, by examining if the established nonlinear relationship between age and prison misconduct varies by sex, especially during the younger age categories.

\subsection{Age, Race/Ethnicity, and Prison Misconduct}

Expanding on the prison misconduct literature, several studies have examined racial and ethnic differences in prison misconduct studies (e.g., Berg \& DeLisi, 2006; DeLisi, 2003; DeLisi et al., 2011; Gover et al., 2008; Harer \& Steffensmeier, 1996; Huebner, 2003; Steiner \& Wooldredge, 2009a; Tasca et al., 2010); however, few studies examine the joint effects of age and race on prison misconduct. Prior research has found differences that exist between whites and blacks (Harer \& Steffensmeier, 1996), between whites and nonwhites (Cao et al., 1997; DeLisi, 2003; DeLisi \& Munoz, 2003), and specifically between whites, blacks, and Latinos (Berg \& DeLisi, 2006; Steiner \& Wooldredge, 2009a) for predicting prison misconduct.

Race/ethnicity differences exist especially when predicting different types of misconduct. For example, prior research has documented that black inmates are significantly more violent in prison as compared to white inmates (Harer \& Steffensmeier, 1996; Kuanliang \& Sorensen, 2008; Steiner \& Wooldredge, 2009a). Further, Hispanic male inmates are more likely to engage in prison assaults or 
violence (Berg \& DeLisis, 2006; Steiner \& Wooldredge, 2009a). Prior research has also found that black and Hispanic inmates are less likely than white inmates to engage in alcohol and/or drug misconduct (Harer \& Steffensmeier 1996; Kuanliang $\&$ Sorensen, 2008; Steiner \& Wooldredge, 2009a).

Only one study has examined the potential conditioning effect of race/ethnicity on the relationship between age and prison misconduct. Innes (1997) explored the interaction effects of age, race, and prison misconduct among federal prisoners using a Tobit analysis. A significant interaction effect was found between age and nonHispanic blacks inmates; thus indicating that non-Hispanic black inmates do not decline uniformly with age. However, since age is measured as a continuous variable, the findings are unable to specify the precise age-misconduct curve for non-Hispanic black inmates. Additional research is warranted to clarify the interaction effect of race/ethnicity on the nonlinear relationship between age and prison misconduct, and further, to see if these results are replicated within a state prisoner sample.

Examining the potential conditioning effect of race/ethnicity on the agemisconduct relationship may further shed light on an inmate's adaptation to prison. According to prior research, the age-misconduct relationship may be more pronounced for black or Latino prisoners if certain subcultural value systems, which condone, call for, and/or require violence, are imported into prison (Harer \& Steffensmeier, 1996; Irwin \& Cressey, 1962; Steiner \& Wooldredge, 2009a). For example, Anderson's (1999) work on the Code of the Street suggests that younger, black offenders may be likely to respond to provocation with violence and to view violence as a necessary way of establishing one's credibility. Prisons are settings in which such a code arguably assumes greater importance. Accordingly, it can be anticipated that younger, black inmates may be more likely to enter prison with such a code, which then may result in more provocation and violence.

Juxtaposed against the above importation argument, an opposing deprivation argument may better explain the conditioning effects of race/ethnicity. Arguments exist which suggest the idea that prison misconduct may instead reflect a potential source of racism within the criminal justice system (Marquart, 1986; Poole \& Regoli, 1980). For example, higher levels of misconduct among black inmates may reflect racial discrimination from correctional officers. Differential treatment from officers, for example, may provoke feelings of resentment and hostility among black inmates that ultimately leads to more disciplinary infractions. Further research suggests that 
correctional officers often have a hostile relationship with younger inmates, and that young offenders, in turn, express hostility toward staff (Bishop \& Frazier, 2000; Scott $\&$ Steinberg, 2008). Against this backdrop, it can be anticipated that the potential for racial discrimination to exist within prisons combined with differential treatment toward young offenders by correctional officers may lead to young, black inmates acting out and engaging in higher levels of misconduct. The current study proposes to extend prior research by examining if racial and ethnic differences condition the age-misconduct relationship, especially for younger inmates.

\section{The Current Study}

The current study examines the potential interaction effects between age and sex, and separately, between age and race/ethnicity (i.e., white, non-Hispanic black, and Hispanic). In particular, the following research questions are examined:

1. To what extent does the age-misconduct relationship vary by sex?

2. To what extent does the age-misconduct relationship vary by race/ethnicity?

\subsection{Data}

The data in the current study includes all inmates admitted to the Florida Department of Corrections (FDOC) from 1995 to $2000(N=137,552)$. The data allows for the systematic investigation of year specific age-dummy variables to examine the precise effect of age on misconduct (age 18, age 19, age 20, etc.). In addition, the large and extensive dataset provides a wealth of information including inmate demographics, prior criminal histories, as well as conduct while in prison (including various types of misconduct).

\subsection{Variables}

The dependent variables in the current study were based on official disciplinarily infraction records and include several types of prison misconduct. Infractions were coded into five categories of prison misconduct based on level of seriousness: all types or general, violent (e.g., assault, threats), property (e.g., theft, vandalism, nondrug contraband), drug (e.g., possession, manufacturing, trafficking), and disorderly (e.g., disobeying orders, unauthorized activity). For each type of misconduct, a count of the number of disciplinary infractions for each inmate was used in the analyses.

The main independent variables include age, sex, and race/ethnicity. Age at admission ranged from 13 to 88 . Age specific dummy variables (e.g., 16 or younger, 
$17,18,19,20$, etc.) were constructed to examine the precise functional form of the age-misconduct relationship. Next, the current study examines white, non-Hispanic black and Hispanic inmates and dichotomous measures $(0=$ no, $1=$ yes $)$ were created for non-Hispanic black (52.18\%) and Hispanic (9.05\%) inmates. The omitted group was white inmates $(38.77 \%)$. Sex is also a dichotomous measure $(1=$ male and $0=$ female) with the majority of inmates $(91.64 \%)$ being male.

The remaining covariates in the analyses include in-prison and criminal background measures. Two in-prison measures are also included in the analyses given that certain circumstances in prison may influence an inmate's conduct. The number of transfers an inmate had while incarcerated was included in the model. The number of transfers ranged from 0 to 93 and the average number of transfers was seven. In addition, a control for the total time-served (in months) in prison was also included in the analysis. The average number of months served in prison was approximately 25 months.

Four criminal history background measures were also included in the analysis. The first is the most serious offense for which inmates were serving time and was categorized as violent or sex, property, drug, or other. For each offense, four dummy variables $(0=$ no; $1=$ yes $)$ were created and included in the analysis. The second is the number of prior Florida prison sentences. The third is the number of prior Florida supervision violations. The final criminal history measure is the number prior convictions for crimes by type (i.e., violent, property, drug, and other).

\subsection{Analytic Strategy}

The current analysis uses negative binomial regression to examine if the relationship between age and prison misconduct varies by sex, and also, if the relationship between age and prison misconduct varies by race/ethnicity (i.e., nonHispanic white, non-Hispanic black, and Hispanic). The current study also employs multiple interaction techniques to unravel the relationship between age, sex, and prison misconduct and further age, race/ethnicity, and prison misconduct. The first set of analyses explores if the relationship between age and prison misconduct varies by sex. Two methods are used to examine possible interaction effects: (1) the spiltsample method, and (2) using multiplicative interaction terms. The split-sample technique divides the sample into subsamples based on sex (i.e., male and female). Next, full negative binomial regression models for each sample will be examined and 
coefficients for each age dummy variable will be obtained. In order to determine whether differences in the negative binomial regression age coefficients were statistically significant, a Z-statistic was calculated using the following formula:

$$
Z=\frac{b_{1}-b_{2}}{\sqrt{ } S E b_{1}^{2}+S E b_{2}^{2}}
$$

Scholars have claimed that the above $\mathrm{Z}$ test is the most appropriate when interested in testing for the equality of regression coefficients (Brame, Paternoster, Mazerolle, \& Piquero, 1998; Paternoster, Brame, Mazerolle, \& Piquero, 1998). ${ }^{1}$

The next method for examining potential interactions effect of sex is to create multiplicative interaction terms to represent the interaction of each age dummy variable and sex, and then include these terms in the negative binomial regression models. A Wald test of joint significance will also be conducted to examine the joint significance of the added interaction terms.

The second part of the analyses will examine if the relationship between age and prison misconduct varies by race/ethnicity. Similar to sex, first a split-sample method will be used to analyze separate negative binomial regression models for all three types of race/ethnicity (i.e., Hispanic, black, and white). Then, a Z-statistic will be conducted to examine the equality of the regression coefficients, using white as the comparison category. ${ }^{2}$ Finally, negative binomial regression models will be examined, which include the multiplicative interaction terms for each age dummy variable and black and also, for each age dummy variable and Hispanic.

\section{Results}

\subsection{Descriptive Statistics}

Descriptive statistics for each variable included in the models, separated by sex, are provided in Table 1. Male inmates have a higher average number of disciplinary infractions, compared to female inmates, across all misconduct categories. Female inmates are, on average, older than male inmates (33 vs. 31 , respectively). The

1 For example in the sex analyses, b1 is the misconduct coefficient for the female sample, b2 is the misconduct coefficient for the male sample, SEb1 is the standard error for the misconduct coefficient in the female sample, and SEb2 is the standard error for the misconduct coefficient in the male sample.

2 For example in the race/ethnicity analyses, b1 is the misconduct coefficient for the black sample, b2 is the misconduct coefficient for the white sample, SEb1 is the standard error for the misconduct coefficient in the black sample, and SEb2 is the standard error for the misconduct coefficient in the white sample. 
Table 1. Descriptive Statistics by Sex

\begin{tabular}{|c|c|c|c|c|c|c|c|c|}
\hline \multirow{3}{*}{ Dependent Variables } & \multicolumn{8}{|c|}{ Sex } \\
\hline & \multicolumn{4}{|c|}{ Male $(N=126,057)$} & \multicolumn{4}{|c|}{ Female $(N=11,495)$} \\
\hline & Mean & S.D. & Min. & Max. & Mean & S.D. & Min. & Max. \\
\hline General Misconduct & 2.98 & 6.04 & 0 & 280 & 2.34 & 5.55 & 0 & 104 \\
\hline Violent Misconduct & 0.39 & 1.24 & 0 & 75 & 0.30 & 1.08 & 0 & 28 \\
\hline Property Misconduct & 0.42 & 1.11 & 0 & 46 & 0.30 & 0.91 & 0 & 16 \\
\hline Drug Misconduct & 0.10 & 0.45 & 0 & 9 & 0.03 & 0.24 & 0 & 10 \\
\hline Disorderly Misconduct & 2.06 & 4.43 & 0 & 184 & 1.71 & 4.22 & 0 & 73 \\
\hline Independent Variable & & & & & & & & \\
\hline Age at Admission (years) & 31.40 & 9.81 & 13.45 & 88.15 & 33.14 & 8.26 & 13.89 & 78.31 \\
\hline Control Variables & & & & & & & & \\
\hline Hispanic & 0.09 & 0.29 & 0 & 1 & 0.05 & 0.21 & 0 & 1 \\
\hline Black & 0.52 & 0.50 & 0 & 1 & 0.51 & 0.50 & 0 & 1 \\
\hline Current Offense: Violent & 0.37 & 0.48 & 0 & 1 & 0.28 & 0.45 & 0 & 1 \\
\hline Current Offense: Property & 0.31 & 0.46 & 0 & 1 & 0.30 & 0.46 & 0 & 1 \\
\hline Current Offense: Drug & 0.24 & 0.43 & 0 & 1 & 0.37 & 0.48 & 0 & 1 \\
\hline Current Offense: Other & 0.08 & 0.27 & 0 & 1 & 0.05 & 0.23 & 0 & 1 \\
\hline Number of Transfers & 7.44 & 6.80 & 0 & 93 & 3.57 & 3.99 & 0 & 43 \\
\hline Number Prior Violent Convictions & 1.31 & 1.59 & 0 & 41 & 0.88 & 1.42 & 0 & 45 \\
\hline Number Prior Property Convictions. & 2.83 & 4.46 & 0 & 112 & 2.96 & 6.03 & 0 & 120 \\
\hline Number Prior Drug Convictions. & 1.49 & 2.45 & 0 & 34 & 2.12 & 3.07 & 0 & 70 \\
\hline Number Prior Other Convictions. & 0.55 & 1.01 & 0 & 22 & 0.42 & 0.83 & 0 & 9 \\
\hline Number Prior FL Prison Sentences & 1.04 & 1.51 & 0 & 14 & 0.71 & 1.16 & 0 & 9 \\
\hline Number Prior Supervision Violations & 1.45 & 1.42 & 0 & 11 & 1.82 & 1.62 & 0 & 11 \\
\hline Time Served (months) & 26.38 & 19.50 & 0.03 & 95.99 & 19.96 & 15.61 & 0.03 & 95.95 \\
\hline
\end{tabular}

majority of both male (52.31\%) and female (50.73\%) inmates are black. The most common current prison sentence for males is violent or sex crimes (36.69\%), followed by property $(30.80 \%)$, drug $(24.41 \%)$, and other $(8.11 \%)$. The most common current prison sentence for female inmates is drug offense (36.70\%), followed by property (29.93\%), violent or sex (27.96\%), and other (5.41\%). Male inmates have a higher average number of transfers (7.44), number of prior Florida prison sentences (1.04) 
and served longer in prison (26.38 months) compared to female inmates. However, female inmates have a higher average number of prior supervision violations compared to male inmates.

Descriptive statistics for all variables, separated by race/ethnicity, are also provided in Table 2. Black inmates have the highest average number of general, violent, and disorderly disciplinary infractions. Further, Hispanic inmates have the highest average number of property and drug disciplinary infractions. The average age for inmates in all race/ethnicity groups ranges around early thirties. White inmates are, on average, the oldest (32.62), followed by Hispanic inmates (31.41), and then black inmates (30.76). The majority of inmates are male for Hispanic (95.68\%), black (91.88\%), and white $(90.39 \%)$ inmates. The most serious convicted offenses, which resulted in the current prison sentence for Hispanic inmates, are violent or sex offenses. The most common current conviction type for black inmates are drug offenses (35.38\%) and, for white inmates were property offenses (39.50\%). Black,

Table 2. Descriptive Statistics by Race/Ethnicity

\begin{tabular}{|c|c|c|c|c|c|c|c|c|c|c|c|c|}
\hline \multirow[b]{3}{*}{ Dependent Variables } & \multicolumn{12}{|c|}{ RACE/ETHNICITY } \\
\hline & \multicolumn{4}{|c|}{ Black $(N=71,775)$} & \multicolumn{4}{|c|}{ Hispanic $(N=12,445)$} & \multicolumn{4}{|c|}{ White $(N=53,332)$} \\
\hline & Mean & S.D. & Min. & Max. & Mean & S.D. & Min. & Max. & Mean & S.D. & Min. & Max. \\
\hline General Misconduct & 3.42 & 6.84 & 0 & 280 & 2.89 & 5.18 & 0 & 71 & 2.26 & 4.78 & 0 & 111 \\
\hline Violent Misconduct & 0.49 & 1.41 & 0 & 65 & 0.38 & 1.06 & 0 & 21 & 0.25 & 0.95 & 0 & 75 \\
\hline Property Misconduct & 0.37 & 1.01 & 0 & 46 & 0.49 & 1.16 & 0 & 22 & 0.44 & 1.19 & 0 & 33 \\
\hline Drug Misconduct & 0.07 & 0.34 & 0 & 10 & 0.15 & 0.55 & 0 & 9 & 0.13 & 0.52 & 0 & 9 \\
\hline Disorderly Misconduct & 2.49 & 5.15 & 0 & 184 & 1.87 & 3.63 & 0 & 56 & 1.45 & 3.27 & 0 & 83 \\
\hline \multicolumn{13}{|l|}{ Independent Variable } \\
\hline Age at Admission (years) & 30.76 & 9.28 & 13.45 & 83.33 & 31.41 & 10.20 & 14.53 & 79.50 & 32.62 & 10.04 & 13.75 & 88.15 \\
\hline \multicolumn{13}{|l|}{ Control Variables } \\
\hline Male & 0.92 & 0.27 & 0 & 1 & 0.96 & 0.20 & 0 & 1 & 0.90 & 0.29 & 0 & 1 \\
\hline Current Offense: Violent & 0.34 & 0.47 & 0 & 1 & 0.41 & 0.49 & 0 & 1 & 0.38 & 0.48 & 0 & 1 \\
\hline Current Offense: Property & 0.24 & 0.43 & 0 & 1 & 0.32 & 0.47 & 0 & 1 & 0.39 & 0.49 & 0 & 1 \\
\hline Current Offense: Drug & 0.35 & 0.48 & 0 & 1 & 0.20 & 0.40 & 0 & 1 & 0.13 & 0.34 & 0 & 1 \\
\hline Current Offense: Other & 0.07 & 0.25 & 0 & 1 & 0.06 & 0.23 & 0 & 1 & 0.10 & 0.30 & 0 & 1 \\
\hline Number of Transfers & 7.13 & 6.64 & 0 & 93 & 7.45 & 6.68 & 0 & 67 & 7.02 & 6.78 & 0 & 80 \\
\hline Number Prior Violent Convictions & 1.38 & 1.63 & 0 & 25 & 1.26 & 1.63 & 0 & 32 & 1.12 & 1.48 & 0 & 45 \\
\hline Number Prior Property Convictions & 2.34 & 3.81 & 0 & 119 & 2.59 & 4.37 & 0 & 75 & 3.57 & 5.47 & 0 & 120 \\
\hline Number Prior Drug Convictions & 2.17 & 2.87 & 0 & 32 & 0.92 & 1.85 & 0 & 28 & 0.84 & 1.83 & 0 & 70 \\
\hline \multirow[t]{2}{*}{ Number Prior Other Convictions } & 0.55 & 0.97 & 0 & 20 & 0.41 & 0.86 & 0 & 22 & 0.56 & 1.06 & 0 & 21 \\
\hline & 1.32 & 1.67 & 0 & 14 & 0.46 & 0.95 & 0 & 10 & 0.72 & 1.21 & 0 & 12 \\
\hline Number Prior Supervision Violations & 1.66 & 1.51 & 0 & 11 & 0.91 & 1.10 & 0 & 10 & 1.38 & 1.36 & 0 & 11 \\
\hline Time Served (months) & 25.98 & 19.30 & 0.03 & 95.99 & 28.73 & 20.05 & 0.03 & 95.82 & 24.98 & 19.02 & 0.03 & 95.99 \\
\hline
\end{tabular}


Table 3. Negative Binomial Regression Models Predicting General Prison Misconduct among Inmates

\begin{tabular}{|c|c|c|c|c|c|c|c|c|c|c|}
\hline \multirow[b]{3}{*}{ Intercept } & \multicolumn{4}{|c|}{ Sex } & \multicolumn{6}{|c|}{ Ethnicity } \\
\hline & \multicolumn{2}{|c|}{ Model 1: Male } & \multicolumn{2}{|c|}{ Model 2: Female } & \multicolumn{2}{|c|}{ Model 3: White } & \multicolumn{2}{|c|}{ Model 4: Black } & \multicolumn{2}{|c|}{ Model 5: Hispanic } \\
\hline & -1.52 & $(0.03)^{* * *}$ & -1.12 & $(0.16)^{* * *}$ & -1.00 & $(0.07)^{* * *}$ & -0.90 & $(0.04)^{* * *}$ & -1.25 & $(0.12)^{* * *}$ \\
\hline Age 17 & -0.13 & $(0.04)^{* *}$ & -0.03 & $(0.20)$ & -0.19 & $(0.08)^{*}$ & -0.10 & $(0.05)^{*}$ & -0.11 & $(0.13)$ \\
\hline Age 18 & -0.28 & $(0.04) * * *$ & -0.03 & $(0.19)$ & -0.33 & $(0.07)^{* * *}$ & -0.24 & $(0.05)^{* * *}$ & -0.28 & $(0.12)^{*}$ \\
\hline Age 19 & -0.47 & $(0.04)^{* * *}$ & -0.36 & $(0.18)^{*}$ & -0.51 & $(0.07)^{* * *}$ & -0.45 & $(0.04)^{* * *}$ & -0.46 & $(0.12)^{* * *}$ \\
\hline Age 20 & -0.63 & $(0.04)^{* * *}$ & -0.53 & $(0.18)^{* *}$ & -0.68 & $(0.07)^{* * *}$ & -0.62 & $(0.04)^{* * *}$ & -0.60 & $(0.12)^{* * *}$ \\
\hline Age 21 & -0.90 & $(0.04)^{* * *}$ & -0.81 & $(0.18)^{* * *}$ & -0.96 & $(0.07)^{* * *}$ & -0.86 & $(0.04)^{* * *}$ & -0.95 & $(0.12)^{* * *}$ \\
\hline Age 22 & -1.05 & $(0.04)^{* * *}$ & -0.97 & $(0.18)^{* * *}$ & -1.13 & $(0.07)^{* * *}$ & -1.02 & $(0.05)^{* * *}$ & -0.95 & $(0.12)^{* * *}$ \\
\hline Age 23 & -1.17 & $(0.04)^{* * *}$ & -1.08 & $(0.17)^{* * *}$ & -1.26 & $(0.07)^{* * *}$ & -1.14 & $(0.05)^{* * *}$ & -1.09 & $(0.12)^{* * *}$ \\
\hline Age 24 & -1.26 & $(0.04)^{* * *}$ & -1.11 & $(0.18)^{* * *}$ & -1.40 & $(0.08)^{* * *}$ & -1.22 & $(0.05)^{* * *}$ & -1.12 & $(0.12)^{* * *}$ \\
\hline Age 25 & -1.35 & $(0.04)^{* * *}$ & -1.48 & $(0.17)^{* * *}$ & -1.47 & $(0.07)^{* * *}$ & -1.31 & $(0.05)^{* * *}$ & -1.36 & $(0.12) * * *$ \\
\hline Age 26 & -1.40 & $(0.04)^{* * *}$ & -1.55 & $(0.17)^{* * *}$ & -1.47 & $(0.08)^{* * *}$ & -1.41 & $(0.05)^{* * *}$ & -1.31 & $(0.12)^{* * *}$ \\
\hline Age 27 & -1.46 & $(0.04)^{* * *}$ & -1.53 & $(0.17)^{* * *}$ & -1.59 & $(0.08)^{* * *}$ & -1.41 & $(0.05)^{* * *}$ & -1.38 & $(0.12) * * *$ \\
\hline Age 28 & -1.51 & $(0.04)^{* * *}$ & -1.63 & $(0.17)^{* * *}$ & -1.64 & $(0.08) * * *$ & -1.48 & $(0.05)^{* * *}$ & -1.47 & $(0.12)^{* * *}$ \\
\hline Age 29 & -1.60 & $(0.04)^{* * *}$ & -1.75 & $(0.17)^{* * *}$ & -1.68 & $(0.08)^{* * *}$ & -1.58 & $(0.05)^{* * *}$ & -1.70 & $(0.13)^{* * *}$ \\
\hline Age 30 & -1.73 & $(0.04)^{* * *}$ & -1.87 & $(0.17)^{* * *}$ & -1.84 & $(0.08)^{* * *}$ & -1.75 & $(0.05)^{* * *}$ & -1.47 & $(0.12)^{* * *}$ \\
\hline Age 31 & -1.69 & $(0.04)^{* * *}$ & -1.81 & $(0.17)^{* * *}$ & -1.83 & $(0.08)^{* * *}$ & -1.65 & $(0.05)^{* * *}$ & -1.67 & $(0.12)^{* * *}$ \\
\hline Age 32 & -1.77 & $(0.04)^{* * *}$ & -1.92 & $(0.17)^{* * *}$ & -1.90 & $(0.08)^{* * *}$ & -1.75 & $(0.05)^{* * *}$ & -1.65 & $(0.13)^{* * *}$ \\
\hline Age 33 & -1.76 & $(0.04)^{* * *}$ & -1.90 & $(0.17)^{* * *}$ & -1.86 & $(0.08)^{* * *}$ & -1.75 & $(0.05)^{* * *}$ & -1.70 & $(0.13)^{* * *}$ \\
\hline Age 34 & -1.82 & $(0.04)^{* * *}$ & -2.05 & $(0.17)^{* * *}$ & -1.95 & $(0.08)^{* * *}$ & -1.84 & $(0.05)^{* * *}$ & -1.60 & $(0.13)^{* * *}$ \\
\hline Age 35 & -1.79 & $(0.04)^{* * *}$ & -2.09 & $(0.17)^{* * *}$ & -2.02 & $(0.08)^{* * *}$ & -1.76 & $(0.05)^{* * *}$ & -1.64 & $(0.13)^{* * *}$ \\
\hline Age 36 & -1.98 & $(0.04)^{* * *}$ & -2.03 & $(0.17)^{* * *}$ & -2.12 & $(0.08)^{* * *}$ & -1.95 & $(0.05)^{* * *}$ & -1.77 & $(0.13)^{* * *}$ \\
\hline Age 37 & -2.00 & $(0.04)^{* * *}$ & -2.06 & $(0.17)^{* * *}$ & -2.24 & $(0.08)^{* * *}$ & -1.92 & $(0.05)^{* * *}$ & -1.75 & $(0.13)^{* * *}$ \\
\hline Age 38 & -2.07 & $(0.04)^{* * *}$ & -2.22 & $(0.17)^{* * *}$ & -2.18 & $(0.08)^{* * *}$ & -2.14 & $(0.05)^{* * *}$ & -1.69 & $(0.13)^{* * *}$ \\
\hline Age 39 & -2.11 & $(0.04)^{* * *}$ & -2.31 & $(0.18)^{* * *}$ & -2.16 & $(0.08)^{* * *}$ & -2.20 & $(0.05) * * *$ & -1.82 & $(0.14)^{* * *}$ \\
\hline Age 40 & -2.16 & $(0.04)^{* * *}$ & -1.97 & $(0.18)^{* * *}$ & -2.23 & $(0.08)^{* * *}$ & -2.15 & $(0.05)^{* * *}$ & -1.86 & $(0.14)^{* * *}$ \\
\hline Age 41 & -2.21 & $(0.05)^{* * *}$ & -2.32 & $(0.19)^{* * *}$ & -2.26 & $(0.08)^{* * *}$ & -2.27 & $(0.06)^{* * *}$ & -1.98 & $(0.14)^{* * *}$ \\
\hline Age 42 & -2.18 & $(0.05)^{* * *}$ & -2.37 & $(0.19)^{* * *}$ & -2.29 & $(0.08)^{* * *}$ & -2.23 & $(0.06)^{* * *}$ & -1.90 & $(0.14)^{* * *}$ \\
\hline Age 43 & -2.23 & $(0.05)^{* * *}$ & -2.48 & $(0.20)^{* * *}$ & -2.37 & $(0.09)^{* * *}$ & -2.27 & $(0.06)^{* * *}$ & -1.95 & $(0.15)^{* * *}$ \\
\hline Age 44 & -2.39 & $(0.05)^{* * *}$ & -2.32 & $(0.19)^{* * *}$ & -2.36 & $(0.09)^{* * *}$ & -2.48 & $(0.07)^{* * *}$ & -2.07 & $(0.15)^{* * *}$ \\
\hline Age 45 & -2.32 & $(0.05)^{* * *}$ & -2.71 & $(0.22)^{* * *}$ & -2.46 & $(0.09)^{* * *}$ & -2.36 & $(0.07)^{* * *}$ & -2.06 & $(0.16)^{* * *}$ \\
\hline Age 46 & -2.50 & $(0.06)^{* * *}$ & -2.46 & $(0.24)^{* * *}$ & -2.44 & $(0.10)^{* * *}$ & -2.67 & $(0.07)^{* * *}$ & -2.16 & $(0.17)^{* * *}$ \\
\hline Age 47 & -2.45 & $(0.06)^{* * *}$ & -2.10 & $(0.23)^{* * *}$ & -2.44 & $(0.10)^{* * *}$ & -2.53 & $(0.08) * * *$ & -2.06 & $(0.17)^{* * *}$ \\
\hline Age 48 & -2.59 & $(0.06)^{* * *}$ & -2.95 & $(0.27)^{* * *}$ & -2.58 & $(0.11)^{* * *}$ & -2.81 & $(0.09)^{* * *}$ & -2.17 & $(0.19)^{* * *}$ \\
\hline Age 49 & -2.49 & $(0.07)^{* * *}$ & -3.08 & $(0.32)^{* * *}$ & -2.57 & $(0.11)^{* * *}$ & -2.63 & $(0.09)^{* * *}$ & -2.15 & $(0.20)^{* * *}$ \\
\hline Age 50 & -2.71 & $(0.07)^{* * *}$ & -2.68 & $(0.29)^{* * *}$ & -2.74 & $(0.11)^{* * *}$ & -2.90 & $(0.11)^{* * *}$ & -2.25 & $(0.20)^{* * *}$ \\
\hline Age 51 & -2.49 & $(0.08)^{* * *}$ & -2.46 & $(0.40)^{* * *}$ & -2.44 & $(0.12)^{* * *}$ & -2.59 & $(0.11)^{* * *}$ & -2.50 & $(0.23)^{* * *}$ \\
\hline Age 52 & -2.55 & $(0.08) * * *$ & -1.59 & $(0.29) * * *$ & -2.38 & $(0.12)^{* * *}$ & -2.72 & $(0.12)^{* * *}$ & -2.21 & $(0.24)^{* * *}$ \\
\hline Age 53 & -2.70 & $(0.09)^{* * *}$ & -2.44 & $(0.33)^{* * *}$ & -2.54 & $(0.13)^{* * *}$ & -3.18 & $(0.15)^{* * *}$ & -2.09 & $(0.25)^{* * *}$ \\
\hline Age 54 & -2.67 & $(0.09)^{* * *}$ & -3.44 & $(0.55)^{* * *}$ & -2.58 & $(0.14)^{* * *}$ & -2.93 & $(0.15)^{* * *}$ & -2.87 & $(0.29)^{* * * *}$ \\
\hline Age 55 & -2.63 & $(0.05)^{* * *}$ & -3.32 & $(0.26)^{* * *}$ & -2.40 & $(0.14)^{* * *}$ & -3.18 & $(0.18)^{* * *}$ & -2.52 & $(0.27)^{* * *}$ \\
\hline Age 56 & --- & --- & --- & --- & -2.12 & $(0.15)^{* * *}$ & -2.63 & $(0.18)^{* * *}$ & -2.49 & $(0.31)^{* * *}$ \\
\hline Age 57 & -- & --- & --- & --- & -2.74 & $(0.18)^{* * *}$ & -2.91 & $(0.22) * * *$ & -1.90 & $(0.31)^{* * *}$ \\
\hline Age 58 & --- & --- & --- & --- & -2.69 & $(0.17)^{* * *}$ & -2.59 & $(0.20)^{* * *}$ & -2.38 & $(0.29)^{* * *}$ \\
\hline Age 59 & --- & --- & --- & -- & -2.64 & $(0.20)^{* * *}$ & -2.90 & $(0.22)^{* * *}$ & -2.63 & $(0.40)^{* * *}$ \\
\hline Age 60 & --- & --- & --- & -- & -2.77 & $(0.20)^{* * *}$ & -3.40 & $(0.31)^{* * *}$ & -2.36 & $(0.33)^{* * *}$ \\
\hline Age 61 & --- & --- & --- & --- & -3.01 & $(0.22)^{* * *}$ & -3.30 & $(0.26)^{* * *}$ & -2.77 & $(0.37)^{* * *}$ \\
\hline Age 62 & --- & --- & --- & -- & -2.76 & $(0.24)^{* * *}$ & -3.47 & $(0.38)^{* * *}$ & -2.21 & $(0.44)^{* * *}$ \\
\hline Age 63 & --- & --- & --- & --- & -2.65 & $(0.27)^{* * *}$ & -3.31 & $(0.34) * * *$ & -3.06 & $(0.54)^{* * *}$ \\
\hline Age 64 & --- & --- & --- & -- & -2.81 & $(0.24)^{* * *}$ & -4.61 & $(0.65)^{* * *}$ & -2.17 & $(0.43)^{* * *}$ \\
\hline Age 65 or higher & --- & --- & --- & -- & -2.63 & $(0.12)^{* * *}$ & -3.13 & $(0.17)^{* * *}$ & -2.53 & $(0.23)^{* * *}$ \\
\hline Male & --- & --- & --- & --- & -0.49 & $(0.02)^{* * *}$ & -0.30 & $(0.02)^{* * *}$ & -0.34 & $(0.06)^{* * *}$ \\
\hline Hispanic & 0.17 & $(0.01)^{* * *}$ & 0.06 & $(0.07)$ & --- & --- & --- & --- & --- & --- \\
\hline Black & 0.28 & $(0.01)^{* * *}$ & 0.09 & $(0.03)^{* *}$ & --- & --- & --- & --- & --- & --- \\
\hline Offense: Property & 0.17 & $(0.01)^{* * *}$ & 0.13 & $(0.05)^{* *}$ & 0.12 & $(0.02)^{* * *}$ & 0.16 & $(0.02)^{* * *}$ & 0.27 & $(0.03)^{* * *}$ \\
\hline Offense: Drug & 0.04 & $(0.01)^{* *}$ & -0.12 & $(0.05)^{*}$ & -0.03 & $(0.03)$ & 0.06 & $(0.02) * * *$ & -0.27 & $(0.05)^{* * *}$ \\
\hline Offense: Other & 0.06 & $(0.02)^{* * *}$ & 0.07 & $(0.08)$ & 0.02 & $(0.03)$ & 0.08 & $(0.02)^{* * *}$ & 0.03 & $(0.06)$ \\
\hline Transfers & 0.03 & $(0.00)^{* * *}$ & 0.04 & $(0.00)^{* * *}$ & 0.04 & $(0.00)^{* * *}$ & 0.03 & $(0.00)^{* * *}$ & 0.03 & $(0.00)^{* * *}$ \\
\hline Violent Convictions & 0.04 & $(0.00)^{* * *}$ & 0.09 & $(0.01)^{* * *}$ & 0.03 & $(0.01)^{* * *}$ & 0.05 & $(0.00)^{* * *}$ & 0.03 & $(0.01)^{* * *}$ \\
\hline Property Convictions & -0.01 & $(0.00)^{* * *}$ & -0.01 & $(0.00)^{* *}$ & -0.01 & $(0.00)^{* * *}$ & -0.01 & $(0.00)^{* * *}$ & -0.01 & $(0.00)$ \\
\hline Drug Convictions & -0.04 & $(0.00)^{* * *}$ & -0.01 & $(0.01)$ & -0.02 & $(0.00)^{* * *}$ & -0.05 & $(0.00)^{* * *}$ & 0.01 & $(0.01)$ \\
\hline Other Convictions & 0.02 & $(0.00)^{* * *}$ & 0.07 & $(0.02)^{* *}$ & 0.03 & $(0.01)^{* * *}$ & 0.01 & $(0.01)^{\dagger}$ & 0.05 & $(0.02)^{* *}$ \\
\hline Prior Prison Sentences & 0.10 & $(0.00)^{* * *}$ & 0.08 & $(0.02)^{* * *}$ & 0.11 & $(0.01)^{* * *}$ & 0.11 & $(0.00)^{* * *}$ & 0.11 & $(0.02)^{* * *}$ \\
\hline Supervision Violations & 0.02 & $(0.00)^{* * *}$ & 0.04 & $(0.01)^{* * *}$ & 0.04 & $(0.01)^{* * *}$ & 0.00 & $(0.00)$ & 0.10 & $(0.01)^{* * *}$ \\
\hline Time Served & $(\operatorname{expc}$ & sure) & (expo & sure) & (expo & sure) & (expo & sure) & $(\exp$ & sure) \\
\hline Model X² & 27440 & $22 * * *$ & 2256. & $31 * * *$ & 10573. & $48^{* * *}$ & 15422. & $45^{* * *}$ & 2578. & $84 * * *$ \\
\hline Pseudo $\mathrm{R}^{2}$ & 0 . & 06 & 0. & & 0.0 & & 0. & 05 & & 05 \\
\hline Nagelkerke $\mathrm{R}^{2}$ & 0. & & 0 . & & 0.1 & & 0. & 20 & & 19 \\
\hline
\end{tabular}

NOTES: ${ }^{\dagger} \mathrm{p} \leq .10 * \mathrm{p} \leq .05 * * \mathrm{p} \leq .01 * * * \mathrm{p} \leq .001$; Negative binomial regression coefficients (with standard errors) are presented. Age 16 or younger is omitted as the reference category. Violence is the omitted category for current offense. --- Indicates the last age dummy variable for misconduct because of the sparse number of inmates engaging in at least one infraction for each age category. 
Hispanic, and white inmates all averaged approximately seven numbers of transfers while incarcerated. Black inmates have the highest average number of prior violent and drug convictions and white inmates have the highest average number of prior property and other convictions. Furthermore, black inmates have the highest number of prior Florida prison sentences and prior supervision violations. Finally, Hispanic inmates served the most time in prison (28.73 months) compared to both black and white inmates.

\subsection{Interaction Effects of Sex}

The first analysis explores whether the relationship between age and prison misconduct varies by sex. Table 3 present negative binomial regression models of general misconduct for male and female inmates. The results show that age is inversely related to misconduct for both male and female inmates. However, younger, female inmates have a higher predicted number of disciplinary infractions per month compared to younger, male inmates. Then, at approximately age 25 the agemisconduct relationship becomes similar for both males and females and gradually declines into adulthood. Nevertheless, an examination of slope differences using the Z-statistic shows no significant difference between male and females.

The predicted values for age across various types of misconduct (i.e., violent, property, drug, and disorderly) for both males and females are presented in Figures 1 through 4 to assist the discussion of these results. Figure 1 illustrates that the agemisconduct curve for violent misconduct varies by sex. The relationship between age and violent misconduct for male inmates suggests that the curvilinearity declines rapidly through adolescence and young adulthood, then a gradual decrease into adulthood, with an eventual tapering out in the thirties. However, the age-violent misconduct curve for female inmates illustrates an increase in late adolescence through age 19, then a rapid decline during young adulthood, and finally a gradual decline into adulthood. Further Z-statistic analyses examining the differences in slopes confirm a statistically significant difference between males and females during the younger years (i.e., approximately age 18 through age 24).

Figure 2 shows that male and female inmates display different age-drug misconduct curves. The relationship between age and drug misconduct for male inmates suggests that the nonlinear effect gradually accelerates through adolescence and young adulthood and then decelerates gradually after age 24. Conversely, the age-drug 
Figs. 1-4. Predicted Number of Disciplinary Infractions per Month for Inmates by Sex

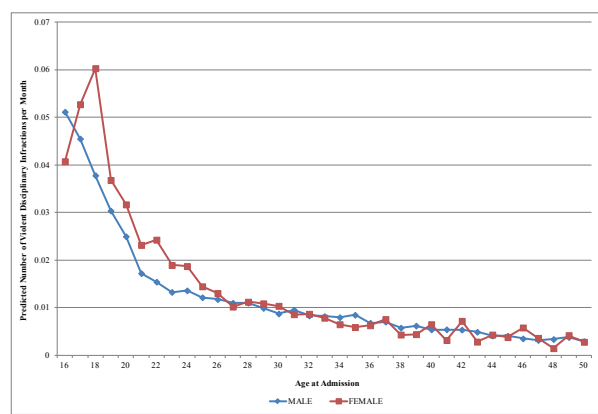

Figure 1: Violent

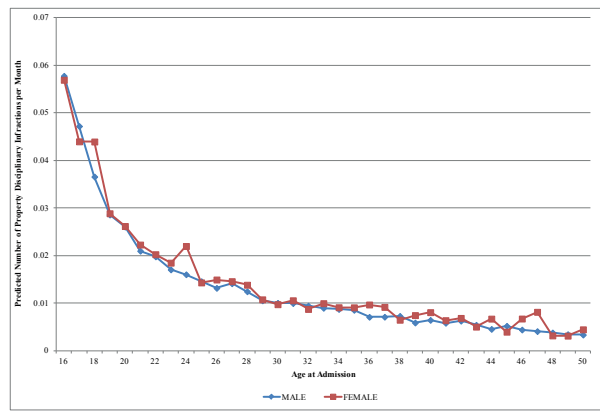

Figure 3: Property

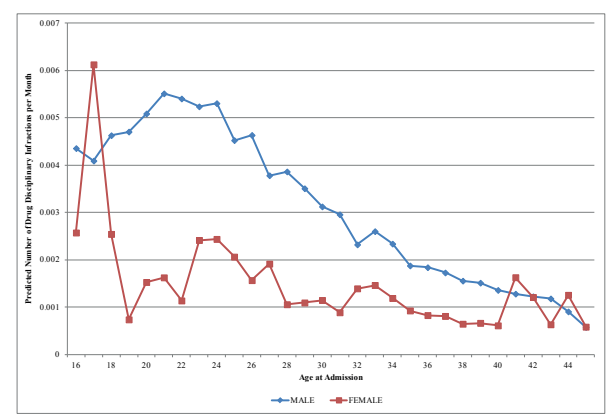

Figure 2: Drug

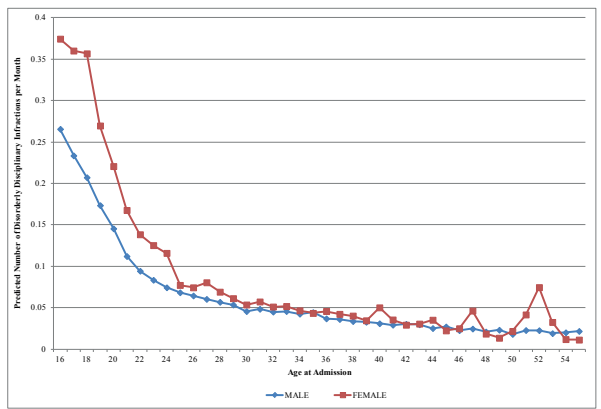

Figure 4: Disorderly

misconduct curve for females accelerates rapidly until approximately age 17 , then drastically decline until age 19, and then, after that, remains relatively stable across all other age categories. Further Z-statistic tests confirm evidence of statistically significant differences between males and females during the younger ages but only for age 19 and age 22. However, it is important to note that drug misconduct is a very rare occurrence and even though the figure is enlarged to display the precise agemisconduct curves the scale, in actuality, is very small. For instance, the drug misconduct curves would appear practically flat when compared to the other misconduct categories.

Figure 3 shows that the relationship between age and property misconduct is parallel for both males and females. Both males and females show similar age trajectories illustrating a consistent negative relationship between age and property misconduct. Further analyses also suggest no statistically significant variation in the relationship between age and property misconduct by sex. 
Finally, Figure 4 presents the age-disorderly misconduct curves for both male and female inmates. Both males and female inmates display a curvilinear relationship; which demonstrates a rapid decline through adolescence and young adulthood, then a gradual decrease into adulthood, with an eventual tapering out in the mid thirties. However, younger, female inmates have a higher predicted number of disorderly infractions per month compared to younger, male inmates. Notwithstanding these results, an examination of slope differences using the Z-statistic shows no statistically significant difference between male and females.

Additional analyses were conducted to examine the conditioning effect of sex. Negative binomial regression models were examined predicting prison misconduct and included several interaction terms (i.e., Age*Sex). The findings showed no considerable differences in the age-misconduct curves between males and females. However, there were some slight differences among the youngest inmates and this was especially true for violent and drug misconduct. For example, there were statistically significant differences between males and females for the youngest inmates (i.e., age 26 and younger) for violent misconduct and a few statistically significant differences among the youngest inmates (i.e., age 17, 19, and 22) for drug misconduct. ${ }^{3}$ Further, robustness checks using a Wald test confirmed the joint significance of all interaction terms (violent: $\chi^{2}=62.21 ; \mathrm{p}=0.002$; drug: $\chi^{2}=60.5$; $\mathrm{p}=0.001)$.

\subsection{Interaction Effects of Race/Ethnicity}

The following set of analyses will examine if the relationship between age and prison misconduct varies by race/ethnicity. Table 3 presents negative binomial regression models for black, Hispanic, and white inmates. Results show that all three racial/ethnic groups display an inverse relationship between age and misconduct. Specifically, young, black inmates have a higher predicted number of disciplinary infractions per month compared to young, white inmates. Conversely, young, Hispanic inmates have a lower predicted number of disciplinary infractions per month compared to young, white inmates. It is not until the late twenties or early thirties until the predicted number of disciplinary infractions become similar for black, Hispanic, and white inmates. A further exploration of slope differences using the Z-statistic demonstrate statistically significant differences for black and white

3 Additional analyses and corresponding tables are available from the author upon request. 
inmates during the late twenties to mid thirties and also statistically significant difference for Hispanic and white inmates during the thirties and forties.

Predicted values for age across various types of misconduct (i.e., violent, property, drug, and disorderly) for black, Hispanic, and white inmates are presented in Figures 5 through 8 to assist the discussion of these results. Figure 5 examines whether the relationship between age and violent misconduct varies by race/ethnicity. All three racial/ethnic groups display a curvilinear relationship between age and misconduct. Black and Hispanic inmates have a higher predicted number of disciplinary infractions per month compared to white inmates, across all age categories. However, this difference is more pronounced for black inmates during the younger age categories. Additional Z-statistic analyses exploring the differences in slopes confirm a small number of statistically significant differences between black and white inmates during the late twenties and mid to late thirties. Additionally, a small number of

Figs. 5-8. Predicted Number of Disciplinary Infractions per Month for Inmates by Race/Ethnicity

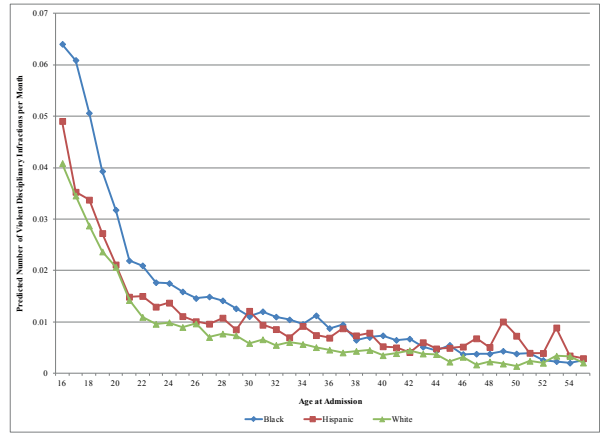

Figure 5: Violent

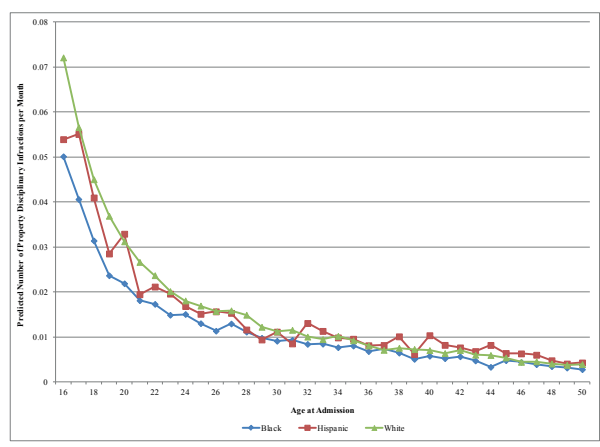

Figure 7: Property

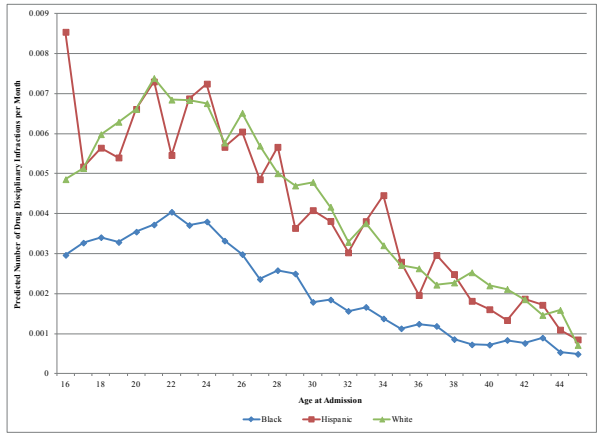

Figure 6: Drug

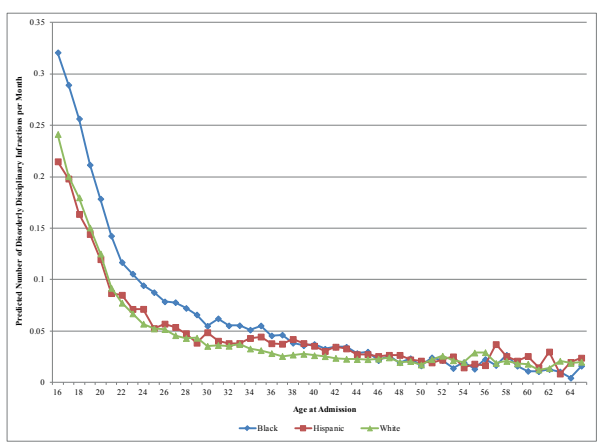

Figure 8: Disorderly 
statistically significant differences between Hispanic and white inmates emerged during the thirties.

Figure 6 illustrates the relationship between age and drug misconduct for all racial and ethnic categories. It is important to reiterate that drug misconduct is a rare occurrence and the noticeable differences in the age-misconduct curves are actually trivial in size. Nevertheless, Hispanic and white inmates illustrate different agemisconduct curves. The age-misconduct curve for white inmates demonstrates a nonlinear effect that gradually accelerates through adolescence and young adulthood and then decelerates gradually thereafter. Yet, the relationship between age and drug misconduct for Hispanic inmates appears almost linear, showing a gradual decrease from age 16 to age 45 . Further Z-statistic tests confirm evidence of statistically significant differences between Hispanic and white inmates. The relationship between age and drug misconduct for black and white inmates is similar to the pattern for all inmates, suggesting that the nonlinear effect gradually accelerates through adolescence and young adulthood and then decelerates gradually after the mid twenties. Further examination of slope differences using the Z-statistic shows no substantial significant difference between black and white inmates

Next, Figure 7 displays the age-property misconduct curves for Hispanic, black, and white inmates. All three racial/ethnic groups display a curvilinear relationship between age and misconduct. However, contrary to the pattern observed for violent misconduct, young, black and young, Hispanic inmates have a lower predicted number of disciplinary infractions per month compared to young, white inmates. In addition, the age-misconduct curve for Hispanic slightly varied during the general decline through late adolescents and into young adulthood. Further Z-statistic tests confirm evidence of statistically significant differences between Hispanic and white inmates for the age twenty dummy variable and also throughout the thirties and forties. Also, a small number of statistically significant differences between black and white inmates emerged during the thirties.

Figure 8 presents the final figure illustrating the relationship between age and disorderly misconduct for Hispanic, black, and white inmates. All inmates display a curvilinear relationship, which demonstrates a rapid decline through adolescence and young adulthood, then a gradual decrease into adulthood. Younger, black inmates have the highest predicted number of disorderly infractions per month compared to younger, white and younger, Hispanic inmates. An examination of slope differences 
using the Z-statistic found evidence of statistically significant differences between black and white inmates during the mid to late twenties and mid thirties. Further, additional tests also found some statistically significant differences for Hispanic and white inmates in the thirties and forties; however, none of these differences demonstrate any substantial differences in the overall age-misconduct curves.

Additional analyses were conducted to explore the potential conditioning effects of race/ethnicity by examining negative binomial regression models predicting various types of prison misconduct which include several interaction terms (i.e., Age*Black, Age*Hispanic). Four key findings can be concluded from these analyses. First, there are no appreciable differences in the age-misconduct curves between black and white inmates. Second, there are some slight differences during the later years for all categories of misconduct, especially drug misconduct; however, these differences do not demonstrate any substantive differences in the misconduct curves. Third, there are no substantive interaction effects between age and Hispanic inmates for violent, property, or disorderly misconduct. Finally, drug misconduct indicates several statistically significant interaction effects for age and Hispanic throughout several age groups from adolescence to adulthood and, thus may indicate different age-misconduct curves as depicted in Figure $6 .{ }^{4}$ Further, robustness checks using a Wald test confirmed joint significance of all interaction terms in all five models (general: $\chi^{2}=339.8 ; \mathrm{p}=0.000$; violent: $\chi^{2}=176.96 ; \mathrm{p}=0.000 ;$ property: $\chi^{2}=117.27$; $\mathrm{p}=0.000$; drug: $\chi^{2}=90.36 ; \mathrm{p}=0.004$; disorderly: $\left.\chi^{2}=355.68 ; \mathrm{p}=0.000\right) .^{5}$

\section{Summary and Discussion}

Several demographic correlates have been associated with prison misconduct. For example, the effect of age on prison misconduct is one of the most consistent and robust determinants of misconduct. In addition, several studies have examined the effect of sex on prison misconduct and also the effect of race/ethnicity on prison misconduct. However, prior research has paid little attention to the conditioning effects of either sex or, separately, race/ethnicity on the age-misconduct relationship.

4 Additional analyses and corresponding tables are available from the author upon request.

5 Additional analyses were examined for all sex models and all race/ethnicity models using logistic regression. Specifically, prison misconduct was quantified as none $(=0)$ or one or more $(=1)$ for all types of misconduct (i.e., violent, property, drug, and disorderly). The results were substantively the same. Furthermore, the goodness-of-fit for the logistic models was evaluated, with and without the interaction effects, using several pseudo R-squareds (i.e., McFadden's, Nagelkerke). A higher value for the interaction models would indicate a better model fit; however, the r-squared values for all models in both the sex and race/ethnicity models remained the same. 
Therefore, the current study attempted to address this deficiency by examining potential interaction effects between age and sex, and separately, between age and race/ethnicity using a large Florida Department of Corrections admission cohort to examine several types of prison misconduct.

The findings from this study clearly indicate that, overall, there are no appreciable differences between males and females when examining the relationship between age and prison misconduct. Further, no substantial differences were found between racial/ ethnic categories. However, younger offenders warrant some attention for their visible differences in levels of misconduct for both males and females and for Hispanic, Black and White inmates. The results also showed slight evidence of variation in the age-misconduct curves by type of misconduct and those results are discussed below.

\subsection{Conditioning Effect of Sex}

The findings suggest that there were no considerable differences between male and female inmates in the predicted age-misconduct curves. However, there were slight to moderate differences among the youngest offenders for violent, drug, and disorderly misconduct. Violent misconduct was the only misconduct type that demonstrated both statistical and substantive significance in the differences between males and females. Several implications flow from these findings. First, studies that examine the relationship between age and misconduct using samples including both males and females should be aware of the potential variation in the age-misconduct curves, especially for younger inmates. In addition, models that do not examine the conditioning effect of sex may be misinterpreting the relationship between age and violent misconduct, especially for females. Future research should also attempt to replicate this finding to see if this variation exists in other state or federal correctional facilities.

Second, these findings support prior work that suggest female inmates are more likely than male inmates to have disciplinary infractions (Cao et al., 1997; Gover et al., 2008). Female inmates have been characterized by some correctional officers as rule breakers and that they are more manipulative and emotional than male inmates. For instance, female inmates have been accused of being more difficult to supervise because of their willingness to argue and lack of respect for authority figures (Pollock, 1986). In addition, female inmates are more likely to have past traumatic relationships, 
less education, and face fewer vocational skills than their male counterparts, which may inadvertently affect their adjustment to prison and makes them less likely to cope with some of the deprivations of prison (Thomas, 2003). Other have further noted that female inmates may be subject to a greater degree of supervision and are often disciplined for delinquent acts that would not apply to males in other facilities, such as using profanity, failing to finish meals, or sharing beauty products (Banks, 2003). However, the reason why females are more likely to receive disciplinary infractions, whether it be that female inmates are more likely engage in misconduct or, conversely, are targeted for stricter punishment, is beyond the scope of the current study.

The current study is also limited by not having direct measures of theories that may help explain the projected age-misconduct curves for both males and females and, thus, future research should attempt to examine some of the above explanations. For instance, Gottfredson and Hirschi's (1990) general theory of crime could be used to examine the potential variation in misconduct among young inmates. If it is truly a "general theory", it should be applicable to both males and females. Therefore, both young male and female inmates may have lower self-control and thus engage in behaviors that are impulsive and risky (i.e., infractions). However, prior research has found self-control to be a significant indicator of infractions for only male inmates (Gover et al., 2008; Hochstetler \& DeLisi, 2005). Future studies should continue to examine theories that may explain why younger, female inmates display higher rates of prison misconduct compared to their male counterparts.

\subsection{Conditioning Effect of Race/Ethnicity}

Similar to sex, there were also no appreciable differences between Hispanic, black, and white inmates in the predicted age-misconduct curves. However, there were slight to moderate differences among the youngest offenders among all four misconduct types. For instance, young, black inmates were more likely to receive violent and disorderly infractions than young, white inmates. Young, black and young, Hispanic inmates were also less likely than young, white inmates to receive a property infraction.

The age-misconduct curves for Hispanic, black, and white inmates did not differ substantively in their trajectories for violent, property, and disorderly misconduct. All three racial/ethnic groups displayed a similar curvilinear relationship between age 
and prison misconduct, which illustrated a rapid decline through adolescence and young adulthood, then a gradual decrease into adulthood. Drug misconduct was the only type of misconduct that demonstrated different trajectories among racial/ethnic groups. For example, the relationship between age and prison misconduct for black and white inmates displayed a nonlinear effect that accelerated through adolescence and young adulthood and then gradually declined into adulthood. Conversely, the relationship between age and drug misconduct for Hispanic inmates appeared almost linear.

Some of the differences found were consistent with prior research. For instance, black inmates were significantly more violent in prison as compared to white inmates (Harer \& Steffensmeier, 1996; Kuanliang \& Sorensen, 2008; Steiner \& Wooldredge, 2009a). Furthermore, black inmates were less likely to have committed a drug or alcohol infraction compared to white inmates (Harer \& Steffensmeier, 1996; Steiner $\&$ Wooldredge, 2009a). Both of these findings were especially true for younger, black inmates. Several implications may be interpreted from these findings. First, the results illustrating that black inmates are less likely to receive a drug infraction compared to white inmates may be too trivial to have any substantive meaning. The predicted count of infractions per month is comparable and, in fact, the curves almost look identical. Drug misconduct, generally, is a rare occurrence due the restriction of drug use in prison. Thus, differences in drug use outside of prison may not reflect the same differences inside prison because certain types of drugs are not as available as they are on the outside (Cope, 2000). Furthermore, the difference in the agemisconduct curves for Hispanic and white inmates may be negligible due to the low occurrence of drug misconduct. Future research should continue to examine if nonlinear relationships between age and drug misconduct are replicated within other correctional facilities.

Turning to violent misconduct, the results of the current study may support arguments which imply that certain subcultural value systems, which condone, call for, and/or require violence, may be imported into prison by minority, particularly Black, inmates (Harer \& Steffensmeier, 1996; Irwin \& Cressey, 1962; Steiner \& Wooldredge, 2009a). Conversely, higher levels of misconduct among black inmates may instead reflect racial bias from correctional officers (Marquart, 1986; Poole \& Regoli, 1980). However, determining if higher levels of violent misconduct resulted from officers reacting unfairly towards inmates, inmates responding illicitly towards 
officers, or inmates targeting each other is beyond the scope of the current study. The current study aimed to identify the precise age-misconduct curves for black, Hispanic, and white inmates and, generally, all inmates displayed similar trajectories. Future research should extend this research by attempting to explain why certain inmates have higher rates of certain types of misconduct than others.

The current study makes significant contributions to the prison misconduct literature by examining the conditioning effects of both race/ethnicity and, separately, sex on the age-misconduct relationship. A better understanding of the type of inmates who are most likely to acquire the most infractions can assist facilities in classification assessments, program structuring, and/or identifying potential unfairness within the prison system. Future research may also want to explore additional conditioning effects, such as sexual orientation, on the age-misconduct relationship to better assess risk, vulnerability, and dangerous behaviors within prison (Pallo, 2018). The importance of maintaining safety and order is a top priority for institutions. Race and ethnicity could be relevant in understanding the dynamics of disorder in prisons. For instance, researchers have implied that certain individuals may be more prone to engage in misconduct because of certain subcultural beliefs they acquired before prison (Harer \& Steffensmeier, 1996; Irwin \& Cressey, 1962; Steiner \& Wooldredge, 2009a). Conversely, others have suggested that certain individuals may be treated unfairly once they enter prison, which in turn, may provoke these individuals to further engage in disorder (Marquart, 1986; Poole \& Regoli, 1980). In addition to race/ethnicity, differences may also exist between males and females. If so, then certain practices used in prison to maintain order may be better tailored for one sex over the other.

\section{References}

Anderson, E. (1999). Code of the street: Decency, violence, and the moral life of the inner city. New York, NY: W.W. Norton and Company.

Bales, W. D., \& Miller, C. H. (2012). The impact of determinate sentencing on prisoner misconduct. Journal of Criminal Justice, 40(5), 394-403.

Banks, C. (2003). Women in prison: A reference handbook. Santa Barbara, CA: ABC-CLIO.

Berg, M. T., \& DeLisi, M. (2006).The correctional melting pot: Race, ethnicity, citizenship, and prison violence. Journal of Criminal Justice, 34, 631-642.

Bishop, D., \& Frazier, C. (2000). Consequences of Transfer. In J. A. Fagan \& F. E. Zimring (Eds.), The changing boarders of juvenile justice: Transfer of adolescents to the criminal court (pp. 227-276). Chicago, IL: University of Chicago Press.

Blackburn, A. G., \& Trulson, C. R. (2010). Sugar and spice and everything nice? Exploring institutional misconduct among serious and violent female delinquents. Journal of Criminal Justice, 38, 1132-1140. 
Brame, R., Paternoster, R., Mazerolle, P., \& Piquero, A. (1998). Testing for the equality of maximum-likelihood regression coefficients between two independent equations. Journal of Quantitative Criminology, 14, 245261.

Cao, L., Zhao, J., \& Dine, S. V. (1997). Prison disciplinary tickets: A test of the deprivation and importation models. Journal of Criminal Justice, 25, 103-113.

Camp, S. D., Gaes, G. G., Langan, N. P., \& Saylor, W. G. (2003). The influence of prisons on inmate misconduct: A multilevel investigation. Justice Quarterly, 20(3), 501-534.

Cope, N. (2000). Drug use in prison: The experience of young offenders. Drugs: Education, Prevention, and Policy, 7, 355-366.

Craddock, A. (1996). A comparative study of male and female prison misconduct careers. The Prison Journal, $76,60-80$.

Cunningham, M. D., \& Sorensen, J. R. (2007). Predictive factors for violent misconduct in close custody. The Prison Journal, 87, 241-253.

DeLisi, M. (2003). Criminal careers behind bars. Behavioral Sciences \& The Law, 21, 653-669.

DeLisi, M., \& Munoz, E. A. (2003). Future dangerousness revisited. Criminal Justice Policy Review, 14, $287-305$.

DeLisi, M., Trulson, C. R., Marquart, J. W., Drury, A. J., \& Kosloski, A. E. (2011). Inside the prison black box: Toward a life course importation model of inmate behavior. International Journal of Offender Therapy and Comparative Criminology, 55, 1186-1207.

Gottfredson, M. R., \& Hirschi, T. (1990). A general theory of crime. Stanford, CA: Stanford University Press.

Gover, A. R., Pérez, D. M., \& Jennings, W. G. (2008). Gender differences in factors contributing to institutional misconduct. The Prison Journal, 88, 378-403.

Griffin, M. L., \& Hepburn, J. R. (2006). The effect of gang affiliation on violent misconduct among inmates during the early years of confinement, Criminal Justice and Behavior, 33, 419-466.

Harer, M. D., \& Steffensmeier, D. J. (1996). Race and prison violence. Criminology, 34, 323-355.

Hochstetler, A., \& DeLisi, M. (2005). Importation, deprivation, and varieties of serving time: An integratedlifestyle-exposure model of prison offending. Journal of Criminal Justice, 33, 257-266.

Houser, K. A., Belenko, S., \& Brennan, P. K. (2012). The effects of mental health and substance abuse disorders on institutional misconduct among female inmates. Justice Quarterly, 29(6), 799-828.

Huebner, B. M. (2003). Administrative determinants of inmate violence: A multilevel analysis. Journal of Criminal Justice, 31, 107-117.

Innes, C. A. (1997). Patterns of misconduct in the federal prison system. Criminal Justice Review, 22, 157-174.

Irwin, J., \& Cressey, D. (1962). Thieves, convicts, and the inmate culture. Social Problems, 10, $142-155$.

Jiang, S., \& Winfree Jr, L. T. (2006). Social support, gender and inmate adjustment to prison life: Insights from a national sample. The Prison Journal, 85, 32-55.

Kuanliang, A., Sorensen, J. R., \& Cunningham, M. D. (2008). Juvenile inmates in an adult prison system: Rate of disciplinary misconduct and violence. Criminal Justice and Behavior, 35, 1186-1201.

Kuanliang, A., \& Sorenson, J. R. (2008). Predictors of self-reported prison misconduct. 21 Criminal Justice Studies: A Critical Journal of Crime, Law, and Society, 21, 27-35.

Marquart, J. W. (1986). Prison guards and the use of physical coercion as a mechanism of prisoner control. Criminology, 24, 347-366.

McClellan, D. S. (1994). Disparity in the discipline of male and female inmates in Texas prisons. Women \& Criminal Justice, 5, 71-97.

McReynolds, L. S., \& Wasserman, G. A. (2008). Risk for disciplinary infractions among incarcerated male youths: Influence of psychiatric disorder. Criminal Justice and Behavior, 35(9), 1174-1185.

McShane, M. D., \& Williams, F. P. (1989). The prison adjustment of juvenile offenders. Crime and Delinquency, $35,254-269$. 
Morris, R. G., Longmire, D. R., Buffington-Vollum, J., \& Vollum, S. (2010). Institutional misconduct and differential parole eligibility among capital inmates. Criminal Justice and Behavior, 37, 417-438.

Pallo, J. (2018). Era of change in Hungarian prison law. Journal of Penal Law and Criminology, 6, 55-71.

Paternoster, R., Brame, R., Mazerolle, P., \& Piquero, A. (1998). Using the correct statistical test for the equality of regression coefficients. Criminology, 36, 859-866.

Pollock, J. M. (1986). Sex and supervision: Guarding male and female inmates. Westport, CT: Greenwood Publishing Group.

Pollock, J. M., Mullings, J. L., \& Crouch, B. M. (2006). Violent women: Findings from the Texas women inmates study. Journal of Interpersonal Violence, 21, 485-502.

Poole, E. D., \& Regoli, R. M. (1980). Race, institutional rule breaking, and institutional response: A study of discretionary decision making in prison. Law and Society Review, 14, 931-946.

Scott, E. S., \& Steinberg, L. (2008). Rethinking juvenile justice. Cambridge, MA: Harvard University Press.

Sorensen, J., \& and Cunningham, M. D. (2008). Conviction offense and prison violence: A comparative study of murderers and other offenders. Crime and Delinquency, 56, 103-125.

Steiner, B. H., \& Butler, D., \& Ellison, J. M. (2014). Causes and correlates of prison inmate misconduct: A systematic review of the evidence. Journal of Criminal Justice, 42, 462-470.

Steiner, B., \& Wooldredge, J. (2008). Inmate versus environmental effects on prison rule violations. Criminal Justice and Behavior, 35, 438-456.

Steiner, B., \& Wooldredge, J. (2009a). The relevance of inmate race/ethnicity versus population composition for understanding prison rule violations. Punishment and Society, 11, 459-489.

Steiner, B., \& Wooldredge, J. (2009b). Individual and environmental effects on assaults and nonviolent rulebreaking by women in prison. Journal of Research in Crime and Delinquency, 46, 437-467.

Tasca, Melinda, Marie L. Griffin and Nancy Rodriguez (2010). The effect of importation and deprivation factors on violent misconduct: An examination of black and Latino youth in prison. Youth Violence and Juvenile Justice, 8, 234-249.

Thomas, J. (2003). Gendered control in prison: The difference difference makes. In B. H. Zaitzow \& J. Thomas (Eds.), Women in prison: Gender and social control (pp. 1-20). Boulder, CO: Lynne Rienner Publishers.

Trulson, C. R., DeLisi, M., \& Marquart, J. W. (2011). Institutional misconduct, delinquent background, and rearrest frequency among serious and violent delinquent offenders. Crime and Delinquency, 57, 709-731.

Valentine, C. L., Mears, D. P., \& Bales, W. D. (2015). Unpacking the relationship between age and prison misconduct. Journal of Criminal Justice, 43, 418-427.

Zingraff, M. T. (1980). Inmate assimilation: A comparison of male and female delinquents. Criminal Justice and Behavior, 7, 275-292. 
